\title{
Well-posedness of the Cauchy problem for a space-dependent anyon Boltzmann equation.
}

\author{
Leif ARKERYD and Anne NOURI \\ Mathematical Sciences, 41296 Göteborg, Sweden, \\ arkeryd@chalmers.se \\ Aix-Marseille University, CNRS, Centrale Marseille, I2M UMR 7373, 13453 Marseille, France, \\ anne.nouri@univ-amu.fr
}

\begin{abstract}
A fully non-linear kinetic Boltzmann equation for anyons is studied in a periodic 1d setting with large initial data. Strong $L^{1}$ solutions are obtained for the Cauchy problem. The main results concern global existence, uniqueness and stabililty.
\end{abstract}

\section{Anyons and the Boltzmann equation.}

Let us first recall the definition of anyon. Consider the wave function $\psi(R, \theta, r, \varphi)$ for two identical particles with center of mass coordinates $(R, \theta)$ and relative coordinates $(r, \varphi)$. Exchanging them, $\varphi \rightarrow \varphi+\pi$, gives a phase factor $e^{2 \pi i}$ for bosons and $e^{\pi i}$ for fermions. In three or more dimensions those are all possibilities. Leinaas and Myrheim proved in 1977 [9], that in one and two dimensions any phase factor is possible in the particle exchange. This became an important topic after the first experimental confirmations in the early 1980-ies, and Frank Wilczek in analogy with the terms bos(e)-ons and fermi-ons coined the name any-ons for the new quasi-particles with any phase. Anyon quasi-particles with e.g. fractional electric charge, have since been observed in various types of experiments.

By moving to a definition in terms of a generalized Pauli exclusion principle, Haldane [8] extended this to a fractional exclusion statistics valid for any dimension, and coinciding with the anyon definition in the one and two dimensional cases. Haldane statistics has also been realized for neutral fermionic atoms at ultra-low temperatures in three dimensions [3]. Wu later derived [17] occupation-number distributions for ideal gases under Haldane statistics by counting states under the new fractional exclusion principle. From the number of quantum states of $N$ identical particles occupying $G$ states being

$$
\frac{(G+N-1) !}{N !(G-1) !} \quad \text { and } \quad \frac{G !}{N !(G-N) !}
$$

in the boson resp. fermion cases, he derived the interpolated number of quantum states for the fractional exclusions to be

$$
\frac{(G+(N-1)(1-\alpha)) !}{N !(G-\alpha N-(1-\alpha)) !}, \quad 0<\alpha<1
$$

\footnotetext{
${ }^{1} 2010$ Mathematics Subject Classification. 82C10, 82C22, 82C40.

${ }^{2}$ Key words; anyon, Haldane statistics, low temperature kinetic theory, quantum Boltzmann equation.
} 
He then obtained for ideal gases the equilibrium statistical distribution

$$
\frac{1}{w\left(e^{(\epsilon-\mu) / T}\right)+\alpha},
$$

where $\epsilon$ denotes particle energy, $\mu$ chemical potential, $T$ temperature, and the function $w(\zeta)$ satisfies

$$
w(\zeta)^{\alpha}(1+w(\zeta))^{1-\alpha}=\zeta \equiv e^{(\epsilon-\mu) / T} .
$$

In particular $w(\zeta)=\zeta-1$ for $\alpha=0$ (bosons) and $w(\zeta)=\zeta$ for $\alpha=1$ (fermions).

In elastic pair collisions, the velocities $\left(v, v_{*}\right)$ before and $\left(v^{\prime}, v_{*}^{\prime}\right)$ after a collision are related by

$$
v^{\prime}=v-n\left[\left(v-v_{*}\right) \cdot n\right], \quad v_{*}^{\prime}=v_{*}+n\left[\left(v-v_{*}\right) \cdot n\right], \quad n \in S^{d-1} .
$$

This preserves mass, linear momentum, and energy in Boltzmann type collision operators. We shall write $f=f(v), \quad f_{*}=f\left(v_{*}\right), \quad f^{\prime}=f\left(v^{\prime}\right), \quad f_{*}^{\prime}=f\left(v_{*}^{\prime}\right)$. An important question for gases with fractional exclusion statistics, is how to calculate their transport properties, in particular how the Boltzmann equation

$$
\partial_{t} f+v \cdot \nabla_{x} f=Q(f)
$$

gets modified. An answer was given by Bhaduri, Bhalerao, and Murthy [2] by generalizing to anyons the filling factors $F(f)$ from the fermion and boson cases, $F(f)=(1+\eta f), \eta=\mp 1$, and by inductive reasoning obtaining as anyon filling factors $F(f)=(1-\alpha f)^{\alpha}(1+(1-\alpha) f)^{1-\alpha}, 0<\alpha<1$.

Namely, with a filling factor $F(f)$ in the collision operator $Q$, the entropy production term becomes

$$
\int Q(f) \log \frac{f}{F(f)} d v
$$

which for equilibrium implies

$$
\frac{f^{\prime}}{F\left(f^{\prime}\right)} \frac{f_{*}^{\prime}}{F\left(f_{*}^{\prime}\right)}=\frac{f}{F(f)} \frac{f_{*}}{F\left(f_{*}\right)} .
$$

Using conservation laws and properties of the Cauchy equation, one concludes that in equilibrium $\frac{f}{F(f)}$ is a Maxwellian. Inserting Wu's equilibrium (1.2) for $f$ and taking the quotient Maxwellian as $e^{-(\epsilon-\mu) / T}$, this gives

$$
f=\frac{1}{w\left(e^{(\epsilon-\mu) / T}\right)+\alpha}, \quad F(f)=f e^{(\epsilon-\mu) / T}=\frac{e^{(\epsilon-\mu) / T}}{w\left(e^{(\epsilon-\mu) / T}\right)+\alpha} .
$$

In particular in the fermion and boson cases,

$$
f=\frac{1}{e^{(\epsilon-\mu) / T}-\eta}, \quad F(f)=\frac{e^{(\epsilon-\mu) / T}}{e^{(\epsilon-\mu) / T}-\eta}, \eta=\mp 1 .
$$

This is consistent with taking an interpolation between the fermion and boson factors as general filling factor, $F(f)=(1-\alpha f)^{\alpha}(1+(1-\alpha) f)^{1-\alpha}, 0<\alpha<1$. It gives the collision operator $Q$ of [2] for Haldane statistics,

$$
Q(f)(v)=Q^{+}(f)-Q^{-}(f)=\int_{\mathbb{R}^{d} \times S^{d-1}} B\left(\left|v-v_{*}\right|, \omega\right)\left[f^{\prime} f_{*}^{\prime} F(f) F\left(f_{*}\right)-f f_{*} F\left(f^{\prime}\right) F\left(f_{*}^{\prime}\right)\right] d v_{*} d \omega .(1.3)
$$

Here $d \omega$ corresponds to the Lebesgue probability measure on the $(d-1)$-sphere. The collision kernel $B(z, \omega)$ in the variables $(z, \omega) \in \mathbb{R}^{d} \times \mathbb{S}^{d-1}$ is positive, locally integrable, and only depends on $|z|$ 
and $|(z, \omega)|$. See [2] for a further discussion of the kernel $B$.

The anyon Boltzmann equation for $0<\alpha<1$ retains important properties from the Fermi-Dirac case, but it has so far not been validated from basic quantum theory. In the filling factor $F(f)=$ $(1-\alpha f)^{\alpha}(1+(1-\alpha) f)^{1-\alpha}, 0<\alpha<1$, the factor $(1-\alpha f)^{\alpha}$ requires the value of $f$ to be between 0 and $\frac{1}{\alpha}$. This is formally preserved by the equation, since the gain term vanishes for $f=\frac{1}{\alpha}$, making the $Q$-term (1.3) and the derivative left hand side of the Boltzmann equation negative there. And the derivative equals the positive gain term for $f=0$, where the loss term vanishes. $F$ is concave with maximum value one at $f=0$ for $\alpha \geq \frac{1}{2}$, and maximum value $\left(\frac{1}{\alpha}-1\right)^{1-2 \alpha}>1$ at $f=\frac{1-2 \alpha}{\alpha(1-\alpha)}$ for $\alpha<\frac{1}{2}$. The collision operator vanishes identically for the equilibrium distribution functions obtained by $\mathrm{Wu}$, but for no other functions.

The Boltzmann equation for the limiting cases, representing boson statistics $(\alpha=0)$ and fermion statistics $(\alpha=1)$, was introduced by Nordheim [15] in 1928. Here the quartic terms in the collision integral cancel, which is used in the analysis. General existence results for the space-homogeneous isotropic boson large data case were obtained in [11, followed by a number of other papers, e.g. [7], [12], [13], 14], and for the space-dependent case near equilibrium in [16]. In the space-dependent fermion case general existence results were obtained in [6] and [10].

For $0<\alpha<1$ there are no cancellations in the collision term. Moreover, the Lipschitz continuity of the collision term is replaced by a weaker Hölder continuity near $f=\frac{1}{\alpha}$. The space-homogeneous initial value problem for the Boltzmann equation with Haldane statistics is

$$
\frac{d f}{d t}=Q(f), \quad f(0, v)=f_{0}(v)
$$

Because of the filling factor $F$, the range for the initial value $f_{0}$ should belong to $\left[0, \frac{1}{\alpha}\right]$, which is also formally preserved by the equation. A good control of $\int f(t, x, v) d v$, which in the spacehomogeneous case is given by the mass conservation, can be used to keep $f$ uniformly away from $\frac{1}{\alpha}$, and $F(f)$ Lipschitz continuous. That was a basic observation behind the existence result for the space-homogeneous anyon Boltzmann equation.

Proposition 1.1 [1] Consider the space-homogeneous equation (1.4) with velocities in $\mathbb{R}^{d}, d \geq 2$ and for hard force kernels with

$$
0<B(z, \theta) \leq C|z|^{\beta}|\sin \theta \cos \theta|^{d-1},
$$

where $\frac{-\pi}{2} \leq \theta \leq \frac{\pi}{2}, 0<\beta \leq 1, d>2$, and $0<\beta<1, d=2$. Let the initial value $f_{0}$ have finite mass and energy. If $0<f_{0} \leq \frac{1}{\alpha}$ and $\operatorname{ess} \sup \left(1+|v|^{s}\right) f_{0}<\infty$ for $s=d-1+\beta$, then the initial value problem for (1.4) has a strong solution in the space of functions continuous from $t \geq 0$ into $L^{1} \cap L^{\infty}$, which conserves mass and energy, and for $t_{0}>0$ given, has ess $\sup _{v, t \leq t_{0}}|v|^{s^{\prime}} f(t, v)$ bounded, where $s^{\prime}=\min \left(s, \frac{2 \beta(d+1)+2}{d}\right)$.

In this proposition, stronger limitations on $B$ would allow for weaker conditions on the initial value $f_{0}$. The proof implies stability; given a sequence of positive initial values $\left(f_{0 n}\right)_{n \in \mathbb{N}}$ with

$$
\sup _{n} \operatorname{ess} \sup f_{0 n}(v)<\frac{1}{\alpha}
$$

and converging in $L^{1}$ to $f_{0}$, there is a subsequence of the solutions converging in $L^{1}$ to a solution with initial value $f_{0}$. 


\section{The main results.}

The present paper considers the space-dependent anyon Boltzmann equation in a slab. Anyons only exist in one and two dimensions. The proof in this paper uses an estimate for the Bony functional in one space dimension, which due to the filling factor $F(f)$, is restricted to the anyon case $v \in \mathbb{R}^{2}$. For $\cos \theta=n \cdot \frac{v-v_{*}}{\left|v-v_{*}\right|}$, the kernel $B\left(\left|v-v_{*}\right|, \theta\right)$ is assumed measurable with

$$
0 \leq B \leq B_{0}
$$

for some $B_{0}>0$. It is also assumed for some $\gamma, \gamma^{\prime}, c_{B}>0$, that

$$
B\left(\left|v-v_{*}\right|, \theta\right)=0 \text { for }|\cos \theta|<\gamma^{\prime}, \quad \text { for } 1-|\cos \theta|<\gamma^{\prime}, \quad \text { and for }\left|v-v_{*}\right|<\gamma,
$$

and that

$$
\int B\left(\left|v-v_{*}\right|, \theta\right) d \theta \geq c_{B}>0 \quad \text { for }\left|v-v_{*}\right| \geq \gamma
$$

The initial datum $f_{0}(x, v)$, periodic in $x$, is assumed to be a measurable function with values in ]0, $\left.\frac{1}{\alpha}\right]$, and such that

$$
\left(1+|v|^{2}\right) f_{0}(x, v) \in L^{1}\left([0,1] \times \mathbb{R}^{2}\right), \int \sup _{x \in[0,1]} f_{0}(x, v) d v=c_{0}<\infty, \inf _{x \in[0,1]} f_{0}(x, v)>0, \text { a.a.v } \in \mathbb{R}^{2} .(2.4
$$

With $v_{1}$ denoting the component of $v$ in the $x$-direction, consider for functions periodic in $x$, the initial value problem

$\partial_{t} f(t, x, v)+v_{1} \partial_{x} f(t, x, v)=Q(f)(t, x, v), \quad f(0, x, v)=f_{0}(x, v), \quad(t, x, v) \in \mathbb{R}_{+} \times[0,1] \times \mathbb{R}^{2}$.

The main result of the present paper is the following theorem.

\section{Theorem 2.1}

There exists a strong solution $f \in \mathcal{C}\left(\left[0, \infty\left[; L^{1}\left([0,1] \times \mathbb{R}^{2}\right)\right)\right.\right.$ of (2.5) with $0<f(t,)<.\frac{1}{\alpha}$ for $t>0$. There is $t_{m}>0$ such that for any $T>t_{m}$, there is $\eta_{T}>0$ so that $f \leq \frac{1}{\alpha}-\eta_{T}$ for $t_{m} \leq t \leq T$.

The solution is unique and stable in the $L^{1}$-norm on each interval of time $[0, T]$.

It conserves mass, first $v$-moments and energy.

\section{$\underline{\text { Remarks. }}$}

The above results seem to be new also in the fermion case where $\alpha=1$.

The approach in the paper can also be used to obtain regularity results.

The control of $\int f(t, x, v) d v$ is in the present space-dependent setting is non-trivial.

The asymptotic behaviour of the solution, not considered in this paper, is related to an entropy for (2.5),

$$
\int\left(f \log f+\left(\frac{1}{\alpha}-f\right) \log (1-\alpha f)^{\alpha}-\left(\frac{1}{1-\alpha}+f\right) \log (1+(1-\alpha) f)^{1-\alpha}\right) d x d p .
$$

An open problem is the behaviour of (2.5) beyond the anyon frame, i.e. for higher $v$-dimensions under Haldane statistics. It seems likely that a close to equilibrium approach as in the classical case, could work with fairly general kernels $B$ for close to equilibrium initial values $f_{0}$ with some regularity and strong decay conditions for large velocities. Any progress on the large data case in 
several space-dimensions under Haldane statistics would be quite interesting.

The lack of Lipschitz continuity of $F(f)$ when $f$ is in a neighborhood of $\frac{1}{\alpha}$ requires some care. Since the gain term vanishes when $f=\frac{1}{\alpha}$ and the derivative becomes negative there, $f$ should start decreasing before reaching this value. The proof that this takes place uniformly over phase-space and approximations, is based on a good control of $\int f(t, x, v) d v$ in the integration of the gain and loss parts of $Q$. That is a main topic in Section 3 together with the study of a family of approximating equations with large velocity cut-off. Based on those results and using the Lipschitz continuity of $F($.$) away from \frac{1}{\alpha}$, in Section 4 contraction mapping techniques prove the well-posedness of the problem, when the initial value $f_{0}$ stays uniformly away from $\frac{1}{\alpha}$. That restriction is removed by a local initial value analysis, which only assumes Hölder continuity of $F($.$) .$

\section{Approximations and control of mass density.}

For any $j \in \mathbb{N}^{*}$, denote by $\psi_{j}$, the cut-off function with

$$
\psi_{j}(r)=0 \quad \text { if } r>j \quad \text { and } \quad \psi_{j}(r)=1 \quad \text { if } r \leq j,
$$

and set

$$
\chi_{j}\left(v, v_{*}, v^{\prime}, v_{*}^{\prime}\right)=\psi_{j}(|v|) \psi_{j}\left(\left|v_{*}\right|\right) \psi_{j}\left(\left|v^{\prime}\right|\right) \psi_{j}\left(\left|v_{*}^{\prime}\right|\right) .
$$

Let $F_{j}$ be defined on $\left[0, \frac{1}{\alpha}\right]$ by

$$
F_{j}(y)=\frac{1-\alpha y}{\left(\frac{1}{j}+1-\alpha y\right)^{1-\alpha}}(1+(1-\alpha) y)^{1-\alpha} .
$$

Denote by $Q_{j}\left(\right.$ resp. $\left.Q_{j}^{+}\right)$, the operator

$$
\begin{aligned}
& Q_{j}(f)(v):=\frac{1}{\pi} \int B\left(\left|v-v_{*}\right|, \theta\right) \chi_{j}\left(v, v_{*}, v^{\prime}, v_{*}^{\prime}\right)\left(f^{\prime} f_{*}^{\prime} F_{j}(f) F_{j}\left(f_{*}\right)-f f_{*} F_{j}\left(f^{\prime}\right) F_{j}\left(f_{*}^{\prime}\right)\right) d v_{*} d \theta, \\
& \text { (resp. its gain part } \left.Q_{j}^{+}(f)(v):=\frac{1}{\pi} \int B\left(\left|v-v_{*}\right|, \theta\right) \chi_{j}\left(v, v_{*}, v^{\prime}, v_{*}^{\prime}\right) f^{\prime} f_{*}^{\prime} F_{j}(f) F_{j}\left(f_{*}\right) d v_{*} d \theta\right) .
\end{aligned}
$$

For $j \in \mathbb{N}^{*}$, let a mollifier $\varphi_{j}$ be defined by $\varphi_{j}(x, v)=j^{3} \varphi(j x, j v)$, where

$$
\varphi \in C_{0}^{\infty}\left(\mathbb{R}^{3}\right), \quad \operatorname{support}(\varphi) \subset[0,1] \times\left\{v \in \mathbb{R}^{2} ;|v| \leq 1\right\}, \quad \varphi \geq 0, \quad \int \varphi(x, v) d x d v=1 .
$$

Let $f_{0, j}$ be the restriction to $[0,1] \times\{v ;|v| \leq j\}$ of $\left(\min \left\{f_{0}, \frac{1}{\alpha}-\frac{1}{j}\right\}\right) * \varphi_{j}$.

The following lemma concerns a corresponding approximation of (2.5).

Lemma 3.1 For $T>0$, there is a unique solution $f_{j} \in C\left([0, T] \times[0,1] ; L^{1}(\{v ;|v| \leq j\})\right)$ to

$$
\partial_{t} f_{j}+v_{1} \partial_{x} f_{j}=Q_{j}\left(f_{j}\right), \quad f_{j}(0, \cdot, \cdot)=f_{0, j}
$$

with values in $\left.] 0, \frac{1}{\alpha}-\eta_{j}\right]$, for some $\eta_{j}>0$. It conserves mass, first moment and energy. 


\section{Proof of Lemma 3.1.}

Let $T>0$ be given. We shall first prove by contraction that for $T_{1}>0$ and small enough, there is a unique solution

$$
f_{\epsilon, j} \in C\left(\left[0, T_{1}\right] \times[0,1] ; L^{1}(\{v ;|v| \leq j\})\right) \cap\left\{f ; f \in\left[0, \frac{1}{\alpha}\right]\right\}
$$

to (3.1). Let the map $\mathcal{C}$ be defined on periodic in $x$ functions in $C\left([0, T] \times[0,1] ; L^{1}(\{v ;|v| \leq\right.$ $j\})) \cap\left\{f ; f \in\left[0, \frac{1}{\alpha}\right]\right\}$ by $\mathcal{C}(f)=g$, where

$\partial_{t} g+v_{1} \partial_{x} g=\frac{1}{\pi}(1-\alpha g)\left(\frac{1+(1-\alpha) f}{\frac{1}{j}+1-\alpha f}\right)^{1-\alpha} \int B \chi_{j} f^{\prime} f_{*}^{\prime} F_{j}\left(f_{*}\right) d v_{*} d \theta-\frac{g}{\pi} \int B \chi_{j} f_{*} F_{j}\left(f^{\prime}\right) F_{j}\left(f_{*}^{\prime}\right) d v_{*} d \theta$,

$g(0, \cdot, \cdot)=f_{0, j}$.

It follows from the linearity of the previous partial differential equation that it has a unique periodic solution $g$ in $C\left([0, T] \times[0,1] ; L^{1}(\{v ;|v| \leq j\})\right)$. For $f$ with values in $\left[0, \frac{1}{\alpha}\right], g$ takes its values in $\left.] 0, \frac{1}{\alpha}\right]$. Indeed, denoting by $g^{\sharp}(t, x, v)=g\left(t, x+t v_{1}, v\right)$,

$$
g^{\sharp}(t, x, v) \geq f_{0, j}\left(x+t v_{1}, v\right) e^{-\int_{0}^{t} \bar{\sigma}_{f}^{\sharp}(r, x, v) d r}>0,
$$

and

$$
\begin{aligned}
(1-\alpha g)^{\sharp}(t, x, v) & =\left(1-\alpha f_{0, j}\right)\left(x+t v_{1}, v\right) e^{-\int_{0}^{t} \tilde{\sigma}_{f}^{\sharp}(r, x, v) d r} \\
& +\frac{\alpha}{\pi} \int_{0}^{t}\left(g \int B \chi_{j} f_{*} F_{j}\left(f^{\prime}\right) F_{j}\left(f_{*}^{\prime}\right) d v_{*} d \theta\right)^{\sharp}(s, x, v) e^{-\int_{s}^{t} \tilde{\sigma}_{f}^{\sharp}(r, x, v) d r} d s \\
& \geq\left(1-\alpha f_{0, j}\right)\left(x+t v_{1}, v\right) e^{-\int_{0}^{t} \tilde{\sigma}_{f}^{\sharp}(r, x, v) d r} \geq 0 .
\end{aligned}
$$

Here,

$$
\begin{aligned}
& \bar{\sigma}_{f}:=\frac{\alpha}{\pi}\left(\frac{1+(1-\alpha) f)}{\frac{1}{j}+1-\alpha f}\right)^{1-\alpha} \int B \chi_{j} f^{\prime} f_{*}^{\prime} F_{j}\left(f_{*}\right) d v_{*} d \theta+\frac{1}{\pi} \int B \chi_{j} f_{*} \tilde{F}_{\epsilon, j}\left(f^{\prime}\right) \tilde{F}_{\epsilon, j}\left(f_{*}^{\prime}\right) d v_{*} d \theta, \\
& \tilde{\sigma}_{f}:=\frac{\alpha}{\pi}\left(\frac{1+(1-\alpha) f)}{\frac{1}{j}+1-\alpha f}\right)^{1-\alpha} \int B \chi_{j} f^{\prime} f_{*}^{\prime} F_{j}\left(f_{*}\right) d v_{*} d \theta .
\end{aligned}
$$

$\mathcal{C}$ is a contraction on $C\left(\left[0, T_{1}\right] \times[0,1] ; L^{1}(\{v ;|v| \leq j\})\right) \cap\left\{f ; f \in\left[0, \frac{1}{\alpha}\right]\right\}$, for $T_{1}>0$ small enough only depending on $j$, since the derivative of the map $F_{j}$ is bounded on $\left[0, \frac{1}{\alpha}\right]$. Let $f_{j}$ be its fixed point, i.e. the solution of (3.1) on $\left[0, T_{1}\right]$. The argument can be repeated and the solution can be continued up to $t=T$. By the exponential form for $f_{j}$ (resp. $1-\alpha f_{j}$ )

$$
f_{j}^{\sharp}(t, x, v) \geq f_{0, j}(x, v) e^{-\int_{0}^{t} \bar{\sigma}_{f_{j}}^{\sharp}(r, x, v) d r}>0, \quad t \in[0, T], x \in[0,1],|v| \leq j,
$$

(resp.

$$
\begin{aligned}
\left(1-\alpha f_{j}\right)^{\sharp}(t, x, v) & \geq\left(1-\alpha f_{0, j}\right)\left(x+t v_{1}, v\right) e^{-\int_{0}^{t} \tilde{\sigma}_{f_{j}}^{\sharp}(r, x, v) d r} \\
& \left.\geq \frac{1}{j e^{c j^{3} T}}, \quad t \in[0, T], x \in[0,1],|v| \leq j\right) .
\end{aligned}
$$

Consequently, for some $\eta_{j}>0$, there is a periodic in $x$ solution $f_{j} \in C\left([0, T] \times[0,1] ; L^{1}(\{v ;|v| \leq j\})\right)$ to (3.1) with values in $\left.] 0, \frac{1}{\alpha}-\eta_{j}\right]$. 
If there were another nonnegative local solution $\tilde{f}_{j}$ to (3.1), defined on $\left[0, T^{\prime}\right]$ for some $\left.\left.T^{\prime} \in\right] 0, T\right]$, then by the exponential form it would stay below $\frac{1}{\alpha}$. The difference $f_{j}-\tilde{f}_{j}$ would for some constant $c_{T^{\prime}}$ satisfy

$$
\int\left|\left(f_{j}-\tilde{f}_{j}\right)^{\sharp}(t, x, v)\right| d x d v \leq c_{T^{\prime}} \int_{0}^{t}\left|\left(f_{j}-\tilde{f}_{j}\right)^{\sharp}(s, x, v)\right| d s d x d v, t \in\left[0, T^{\prime}\right], \quad\left(f_{j}-\tilde{f}_{j}\right)^{\sharp}(0, x, v)=0,
$$

implying that the difference would be identically zero on $\left[0, T^{\prime}\right]$. Thus $f_{j}$ is the unique solution on $[0, T]$ to (3.1), and has its range contained in $\left.] 0, \frac{1}{\alpha}-\eta_{j}\right]$.

Moreover, $f_{j} \in W^{1,1}\left([0, T] \times[0,1] ; L^{1}(\{v ;|v| \leq j\})\right)$. Indeed, $\partial_{x} f_{j}$ satisfies

$$
\begin{array}{r}
\partial_{t}\left(\partial_{x} f_{j}\right)+v_{1} \partial_{x}\left(\partial_{x} f_{j}\right)+\sigma_{j} \partial_{x} f_{j}=\frac{1}{\pi}\left(1-\alpha f_{j}\right) \partial_{x}\left(\left(\frac{1+\left(1-\alpha f_{j}\right)}{\frac{1}{j}+1-\alpha f_{j}}\right)^{1-\alpha} \int B \chi_{j} f_{j}^{\prime} f_{j *}^{\prime} F_{j}\left(f_{j *}\right) d v_{*} d \theta\right) \\
\quad-\frac{f_{j}}{\pi} \partial_{x} \int B \chi_{j} f_{j *} F_{j}\left(f_{j}^{\prime}\right) F_{j}\left(f_{j *}^{\prime}\right) d v_{*} d \theta, \\
\partial_{x} f_{j}(0, \cdot, \cdot)=\partial_{x} f_{0, j},
\end{array}
$$

where

$$
\sigma_{j}:=\frac{\alpha}{\pi}\left(\frac{1+\left(1-\alpha f_{j}\right)}{\frac{1}{j}+1-\alpha f_{j}}\right)^{1-\alpha} \int B \chi_{j} f_{j}^{\prime} f_{j *}^{\prime} F_{j}\left(f_{j *}\right) d v_{*} d \theta+\frac{1}{\pi} \int B \chi_{j} f_{j *} F_{j}\left(f_{j}^{\prime}\right) F_{j}\left(f_{j *}^{\prime}\right) d v_{*} d \theta .
$$

Using the exponential form of $\partial_{x} f_{j}$, multiplying it by $\operatorname{sgn}\left(\partial_{x} f_{j}\right)$, integrating the resulting equation on $[0,1] \times\{v ;|v| \leq j\}$ and using a Gronwall argument leads to a $j$-dependent bound for $\int\left|\partial_{x} f_{j}(t, x, v)\right| d x d v$ on $[0, T]$. Hence, also from (3.1) and the bounded domain of integration of $v$, $\partial_{t} f_{j}$ also belongs to $L^{\infty}\left(0, T ; L^{1}([0,1] \times\{v ;|v| \leq j\})\right)$.

The remaining part of this section is devoted to obtaining a uniform control with respect to $j \in \mathbb{N}^{*}$ of

$$
\int \sup _{t>0, x \in[0,1]} f_{j}^{\sharp}(t, x, v) d v .
$$

It relies on the following four lemmas, where the first is an estimate of the Bony functionals,

$$
\bar{B}_{j}(t):=\int_{0}^{1} \int\left|v-v_{*}\right|^{2} B \chi_{j} f_{j} f_{j *} F_{j}\left(f_{j}^{\prime}\right) F_{j}\left(f_{j *}^{\prime}\right) d v d v_{*} d \theta d x, \quad t \geq 0 .
$$

\section{Lemma 3.2}

For $T>0$ it holds that

$$
\int_{0}^{T} \bar{B}_{j}(t) d t \leq c_{0}^{\prime}(1+T), \quad j \in \mathbb{N}^{*},
$$

with $c_{0}^{\prime}$ only depending on $\int f_{0}(x, v) d x d v$ and on $\int|v|^{2} f_{0}(x, v) d x d v$.

\section{Proof of Lemma 3.2.}

Denote $f_{j}$ by $f$ for simplicity. The proof is an extension of the classical one (cf [4, [5]), as follows. The integral over time of the momentum $\int v_{1} f(t, 0, v) d v$ (resp. the momentum flux 
$\left.\int v_{1}^{2} f(t, 0, v) d v\right)$ is first controlled. Let $\beta \in C^{1}([0,1])$ be such that $\beta(0)=-1$ and $\beta(1)=1$. Multiply (3.1) by $\beta(x)$ (resp. $v_{1} \beta(x)$ ) and integrate over $[0, t] \times[0,1] \times \mathbb{R}^{2}$. It gives

$$
\begin{aligned}
\int_{0}^{t} \int v_{1} f(\tau, 0, v) d v d \tau=\frac{1}{2}\left(\int \beta(x) f_{0}(x, v) d x d v\right. & -\int \beta(x) f(t, x, v) d x d v \\
& \left.+\int_{0}^{t} \int \beta^{\prime}(x) v_{1} f(\tau, x, v) d x d v d \tau\right)
\end{aligned}
$$

(resp.

$$
\begin{aligned}
\int_{0}^{t} \int v_{1}^{2} f(\tau, 0, v) d v d \tau=\frac{1}{2}\left(\int \beta(x) v_{1} f_{0}(x, v) d x d v\right. & -\int \beta(x) v_{1} f(t, x, v) d x d v \\
& \left.\left.+\int_{0}^{t} \int \beta^{\prime}(x) v_{1}^{2} f(\tau, x, v) d x d v d \tau\right)\right) .
\end{aligned}
$$

Consequently, using the conservation of mass and energy of $f$,

$$
\left|\int_{0}^{t} \int v_{1} f(\tau, 0, v) d v d \tau\right|+\int_{0}^{t} \int v_{1}^{2} f(\tau, 0, v) d v d \tau \leq c(1+t)
$$

Let

$$
\mathcal{I}(t)=\int_{x<y}\left(v_{1}-v_{* 1}\right) f(t, x, v) f\left(t, y, v_{*}\right) d x d y d v d v_{*} .
$$

It results from

$\mathcal{I}^{\prime}(t)=-\int\left(v_{1}-v_{* 1}\right)^{2} f(t, x, v) f\left(t, x, v_{*}\right) d x d v d v_{*}+2 \int v_{* 1}\left(v_{* 1}-v_{1}\right) f\left(t, 0, v_{*}\right) f(t, x, v) d x d v d v_{*}$, and the conservations of the mass, momentum and energy of $f$ that

$$
\begin{aligned}
& \int_{0}^{t} \int_{0}^{1} \int\left(v_{1}-v_{* 1}\right)^{2} f(s, x, v) f_{*}\left(s, x, v_{*}\right) d v d v_{*} d x d s \\
& \leq 2 \int f_{0}(x, v) d x d v \int\left|v_{1}\right| f_{0}(x, v) d v+2 \int f(t, x, v) d x d v \int\left|v_{1}\right| f(t, x, v) d x d v \\
& +2 \int_{0}^{t} \int v_{* 1}\left(v_{* 1}-v_{1}\right) f\left(\tau, 0, v_{*}\right) f(\tau, x, v) d x d v d v_{*} d \tau \\
& \leq 2 \int f_{0}(x, v) d x d v \int\left(1+|v|^{2}\right) f_{0}(x, v) d v+2 \int f(t, x, v) d x d v \int\left(1+|v|^{2}\right) f(t, x, v) d x d v \\
& +2 \int_{0}^{t}\left(\int v_{* 1}^{2} f\left(\tau, 0, v_{*}\right) d v_{*}\right) d \tau \int f_{0}(x, v) d x d v-2 \int_{0}^{t}\left(\int v_{* 1} f\left(\tau, 0, v_{*}\right) d v_{*}\right) d \tau \int v_{1} f_{0}(x, v) d x d v \\
& \leq c\left(1+\int_{0}^{t} \int v_{1}^{2} f(\tau, 0, v) d v d \tau+\left|\int_{0}^{t} \int v_{1} f(\tau, 0, v) d v\right|\right) .
\end{aligned}
$$

And so, by (3.4),

$$
\int_{0}^{t} \int_{0}^{1} \int\left(v_{1}-v_{* 1}\right)^{2} f(\tau, x, v) f\left(\tau, x, v_{*}\right) d x d v d v_{*} d \tau \leq c(1+t) .
$$


Here, $c$ is a constant depending only on $\int f_{0}(x, v) d x d v$ and $\int|v|^{2} f_{0}(x, v) d x d v$. Denote by $u_{1}=\frac{\int v_{1} f d v}{\int f d v}$. It holds

$$
\begin{aligned}
\int_{0}^{t} \int_{0}^{1} \int\left(v_{1}-u_{1}\right)^{2} B \chi_{j} f f_{*} & F_{j}\left(f^{\prime}\right) F_{j}\left(f_{*}^{\prime}\right)\left(s, x, v, v_{*}, \theta\right) d v d v_{*} d \theta d x d s \\
& \leq c \int_{0}^{t} \int_{0}^{1} \int\left(v_{1}-u_{1}\right)^{2} f f_{*}\left(s, x, v, v_{*}\right) d v d v_{*} d x d s \\
& =\frac{c}{2} \int_{0}^{t} \int_{0}^{1} \int\left(v_{1}-v_{* 1}\right)^{2} f f_{*}\left(s, x, v, v_{*}\right) d v d v_{*} d x d s \\
& \leq c(1+t) .
\end{aligned}
$$

Multiply equation (3.1) for $f$ by $v_{1}^{2}$, integrate and use that $\int v_{1}^{2} Q_{j}(f) d v=\int\left(v_{1}-u_{1}\right)^{2} Q_{j}(f) d v$ and (3.6). It results

$$
\begin{aligned}
& \frac{1}{\pi} \int_{0}^{t} \int\left(v_{1}-u_{1}\right)^{2} B \chi_{j} f^{\prime} f_{*}^{\prime} F_{j}(f) F_{j}\left(f_{*}\right) d v d v_{*} d \theta d x d s \\
& =\int v_{1}^{2} f(t, x, v) d x d v-\int v_{1}^{2} f_{0}(x, v) d x d v+\frac{1}{\pi} \int_{0}^{t} \int\left(v_{1}-u_{1}\right)^{2} B \chi_{j} f f_{*} F_{j}\left(f^{\prime}\right) F_{j}\left(f_{*}^{\prime}\right) d x d v d v_{*} d \theta d s \\
& <c_{0}(1+t),
\end{aligned}
$$

where $c_{0}$ is a constant only depending on $\int f_{0}(x, v) d x d v$ and $\int|v|^{2} f_{0}(x, v) d x d v$.

After a collision transform the left hand side can be written

$$
\begin{aligned}
& \frac{1}{\pi} \int_{0}^{t} \int\left(v_{1}^{\prime}-u_{1}\right)^{2} B \chi_{j} f f_{*} F_{j}\left(f^{\prime}\right) F_{j}\left(f_{*}^{\prime}\right) d v d v_{*} d \theta d x d s \\
& =\frac{1}{\pi} \int_{0}^{t} \int\left(c_{1}-n_{1}\left[\left(v-v_{*}\right) \cdot n\right]\right)^{2} B \chi_{j} f f_{*} F_{j}\left(f^{\prime}\right) F_{j}\left(f_{*}^{\prime}\right) d v d v_{*} d \theta d x d s,
\end{aligned}
$$

where $c_{1}=v_{1}-u_{1}$. Expand $\left(c_{1}-n_{1}\left[\left(v-v_{*}\right) \cdot n\right]\right)^{2}$, and remove the positive term containing $c_{1}^{2}$.

The term containing $n_{1}^{2}\left[\left(v-v_{*}\right) \cdot n\right]^{2}$ is estimated from below. When $n$ is replaced by an orthogonal (direct) unit vector $n_{\perp}, v^{\prime}$ and $v_{*}^{\prime}$ are shifted and the product $f f_{*} F_{j}\left(f^{\prime}\right) F_{j}\left(f_{*}^{\prime}\right)$ is unchanged. In $\mathbb{R}^{2}$ the ratio between the sum of the integrand factors $n_{1}^{2}\left[\left(v-v_{*}\right) \cdot n\right]^{2}+n_{\perp 1}^{2}\left[\left(v-v_{*}\right) \cdot n_{\perp}\right]^{2}$ and $\left|v-v_{*}\right|^{2}$, is, outside of the angular cut-off (2.2), uniformly bounded from below by $\gamma^{\prime 2}$. Indeed, if $\alpha$ denotes the angle between $\frac{v-v_{*}}{\left|v-v_{*}\right|}$ and $n$,

$$
\begin{aligned}
n_{1}^{2}\left[\frac{v-v_{*}}{\left|v-v_{*}\right|} \cdot n\right]^{2}+n_{\perp 1}^{2}\left[\frac{v-v_{*}}{\left|v-v_{*}\right|} \cdot n_{\perp}\right]^{2} & =\cos ^{2} \theta \cos ^{2} \alpha+\sin ^{2} \theta \sin ^{2} \alpha \\
& \geq \gamma^{\prime 2} \cos ^{2} \alpha+\gamma^{\prime}\left(2-\gamma^{\prime}\right) \sin ^{2} \alpha \\
& \geq \gamma^{\prime 2}, \quad \gamma^{\prime}<|\cos \theta|<1-\gamma^{\prime}, \quad \alpha \in[0,2 \pi] .
\end{aligned}
$$

This is where the condition $v \in \mathbb{R}^{2}$ is used.

That leads to the lower bound

$$
\begin{aligned}
& \int_{0}^{t} \int n_{1}^{2}\left[\left(v-v_{*}\right) \cdot n\right]^{2} B \chi_{j} f f_{*} F_{j}\left(f^{\prime}\right) F_{j}\left(f_{*}^{\prime}\right) d v d v_{*} d \theta d x d s \\
& \geq \gamma^{\prime 2} \int_{0}^{t} \int\left|v-v_{*}\right|^{2} B \chi_{j} f f_{*} F_{j}\left(f^{\prime}\right) F_{j}\left(f_{*}^{\prime}\right) d v d v_{*} d \theta d x d s .
\end{aligned}
$$


And so,

$$
\begin{aligned}
& \gamma^{\prime 2} \int_{0}^{t} \int\left|v-v_{*}\right|^{2} B \chi_{j} f f_{*} F_{j}\left(f^{\prime}\right) F_{j}\left(f_{*}^{\prime}\right) d v d v_{*} d \theta d x d s \\
& \leq c_{0}(1+t)+2 \int_{0}^{t} \int\left(v_{1}-u_{1}\right) n_{1}\left[\left(v-v_{*}\right) \cdot n\right] B \chi_{j} f f_{*} F_{j}\left(f^{\prime}\right) F_{j}\left(f_{*}^{\prime}\right) d v d v_{*} d \theta d x d s \\
& \leq c_{0}(1+t)+2 \int_{0}^{t} \int\left(v_{1}\left(v_{2}-v_{* 2}\right) n_{1} n_{2}\right) B \chi_{j} f f_{*} F_{j}\left(f^{\prime}\right) F_{j}\left(f_{*}^{\prime}\right) d v d v_{*} d \theta d x d s
\end{aligned}
$$

since

$$
\begin{aligned}
& \int u_{1}\left(v_{1}-v_{* 1}\right) n_{1}^{2} B \chi_{j} f f_{*} F_{j}\left(f^{\prime}\right) F_{j}\left(f_{*}^{\prime}\right) d v d v_{*} d \theta d x \\
& =\int u_{1}\left(v_{2}-v_{* 2}\right) n_{1} n_{2} \chi_{j} B f f_{*} F\left(i f^{\prime}\right) F_{j}\left(f_{*}^{\prime}\right) d v d v_{*} d \theta d x=0,
\end{aligned}
$$

by an exchange of the variables $v$ and $v_{*}$. Moreover, exchanging first the variables $v$ and $v_{*}$,

$$
\begin{aligned}
2 \int_{0}^{t} \int v_{1}\left(v_{2}-v_{* 2}\right) n_{1} n_{2} B \chi_{j} f f_{*} F_{j}\left(f^{\prime}\right) F_{j}\left(f_{*}^{\prime}\right) d v d v_{*} d \theta d x d s \\
=\int_{0}^{t} \int\left(v_{1}-v_{* 1}\right)\left(v_{2}-v_{* 2}\right) n_{1} n_{2} B \chi_{j} f f_{*} F_{j}\left(f^{\prime}\right) F_{j}\left(f_{*}^{\prime}\right) d v d v_{*} d \theta d x d s \\
\leq \leq \frac{1}{\gamma^{\prime 2}} \int_{0}^{t} \int\left(v_{1}-v_{* 1}\right)^{2} n_{1}^{2} B \chi_{j} f f_{*} F_{j}\left(f^{\prime}\right) F_{j}\left(f_{*}^{\prime}\right) d v d v_{*} d \theta d x d s \\
\quad+\frac{\gamma^{\prime 2}}{4} \int_{0}^{t} \int\left(v_{2}-v_{* 2}\right)^{2} n_{2}^{2} B \chi_{j} f f_{*} F_{j}\left(f^{\prime}\right) F_{j}\left(f_{*}^{\prime}\right) d v d v_{*} d \theta d x d s \\
\leq \frac{c_{0}}{\gamma^{\prime 2}}(1+t)+\frac{\gamma^{\prime 2}}{4} \int_{0}^{t} \int\left(v_{2}-v_{* 2}\right)^{2} n_{2}^{2} B \chi_{j} f f_{*} F_{j}\left(f^{\prime}\right) F_{j}\left(f_{*}^{\prime}\right) d v d v_{*} d \theta d x d s .
\end{aligned}
$$

It follows that

$$
\int_{0}^{t} \int\left|v-v_{*}\right|^{2} B \chi_{j} f f_{*} F_{j}\left(f^{\prime}\right) F_{j}\left(f_{*}^{\prime}\right) d v d v_{*} d \theta d x d s \leq c_{0}^{\prime}(1+t),
$$

with $c_{0}^{\prime}$ only depending on $\int f_{0}(x, v) d x d v$ and $\int|v|^{2} f_{0}(x, v) d x d v$. This completes the proof of the lemma.

\section{Lemma 3.3}

There exist constants $c_{1}^{\prime}$ and $c_{2}^{\prime}$ only depending on $\int f_{0}(x, v) d x d v$ and on $\int|v|^{2} f_{0}(x, v) d x d v$, so that

$$
\int \sup _{0 \leq t \leq T} f_{j}^{\sharp}(t, x, v) d x d v<c_{1}^{\prime}+c_{2}^{\prime} T, \quad j \in \mathbb{N}^{*} \quad T>0 .
$$

Proof of Lemma 3.3.

Denote $f_{j}$ by $f$ for simplicity. Since

$$
f^{\sharp}(t, x, v)=f_{0}(x, v)+\int_{0}^{t} Q_{j}(f)\left(s, x+s v_{1}, v\right) d s,
$$


it holds that

$$
\sup _{0 \leq t \leq T} f^{\sharp}(t, x, v) \leq f_{0}(x, v)+\int_{0}^{T} Q_{j}^{+}(f)\left(t, x+t v_{1}, v\right) d t .
$$

Integrating (3.7) with respect to $(x, v)$ and using Lemma 3.2, gives

$$
\begin{aligned}
\int \sup _{0 \leq t \leq T} f^{\sharp}(t, x, v) d x d v & \leq \int f_{0}(x, v) d x d v+\frac{1}{\pi} \int_{0}^{T} \int B \chi_{j} \\
f\left(t, x+t v_{1}, v^{\prime}\right) f\left(t, x+t v_{1}, v_{*}^{\prime}\right) & F_{j}(f)\left(t, x+t v_{1}, v\right) F_{j}(f)\left(t, x+t v_{1}, v_{*}\right) d v d v_{*} d \theta d x d t \\
& \leq \int f_{0}(x, v) d x d v+\frac{1}{\gamma^{2}} \int_{0}^{T} \int B \chi_{j}\left|v-v_{*}\right|^{2} \\
f\left(t, x, v^{\prime}\right) f\left(t, x, v_{*}^{\prime}\right) & F_{j}(f)(t, x, v) F_{j}(f)\left(t, x, v_{*}\right) d v d v_{*} d \theta d x d t \\
& \leq \int f_{0}(x, v) d x d v+\frac{C_{1}+C_{2} T}{\gamma^{2}} .
\end{aligned}
$$

\section{Lemma 3.4}

Given $T>0$ and $\delta_{1}>0$, there exist $\delta_{2}>0$ and $t_{0}>0$, only depending on $\int f_{0}(x, v) d x d v$ and on $\int|v|^{2} f_{0}(x, v) d x d v$, such that for $t \leq T$

$$
\sup _{x_{0} \in[0,1]} \int_{\left|x-x_{0}\right|<\delta_{2}} \sup _{t \leq s \leq t+t_{0}} f_{j}^{\sharp}(s, x, v) d x d v<\delta_{1}, \quad j \in \mathbb{N}^{*} .
$$

Proof of Lemma 3.4 .

Denote $f_{j}$ by $f$ for simplicity. For $t \leq s \leq t+t_{0}$ it holds,

$$
\begin{aligned}
f^{\sharp}(s, x, v) & =f^{\sharp}\left(t+t_{0}, x, v\right)-\int_{s}^{t+t_{0}} Q_{j}(f)\left(\tau, x+\tau v_{1}, v\right) d \tau \\
& \leq f^{\sharp}\left(t+t_{0}, x, v\right)+\int_{s}^{t+t_{0}} Q_{j}^{-}(f)\left(\tau, x+\tau v_{1}, v\right) d \tau .
\end{aligned}
$$

And so

$$
\sup _{t \leq s \leq t+t_{0}} f^{\sharp}(s, x, v) \leq f^{\sharp}\left(t+t_{0}, x, v\right)+\int_{t}^{t+t_{0}} Q_{j}^{-}(f)\left(s, x+s v_{1}, v\right) d s .
$$


Integrating with respect to $(x, v)$, using Lemma 3.2 and the bound $\frac{1}{\alpha}$ from above of $f$, gives

$$
\begin{aligned}
& \int_{\left|x-x_{0}\right|<\delta_{2}} \sup _{t \leq s \leq t+t_{0}} f^{\sharp}(s, x, v) d x d v \\
& \leq \int_{\left|x-x_{0}\right|<\delta_{2}} f^{\sharp}\left(t+t_{0}, x, v\right) d x d v \\
& +\frac{1}{\pi} \int_{t}^{t+t_{0}} \int B \chi_{j} f^{\sharp}(s, x, v) f\left(s, x+s v_{1}, v_{*}\right) F_{j}(f)\left(s, x+s v_{1}, v^{\prime}\right) F_{j}(f)\left(s, x+s v_{1}, v_{*}^{\prime}\right) d v d v_{*} d \theta d x d s \\
& \leq \int_{\left|x-x_{0}\right|<\delta_{2}} f^{\sharp}\left(t+t_{0}, x, v\right) d x d v+\frac{1}{\lambda^{2}} \int_{t}^{t+t_{0}} \int_{\left|v-v_{*}\right| \geq \lambda} B \chi_{j}\left|v-v_{*}\right|^{2} f^{\sharp}(s, x, v) f\left(s, x+s v_{1}, v_{*}\right) \\
& F_{j}(f)\left(s, x+s v_{1}, v^{\prime}\right) F_{j}(f)\left(s, x+s v_{1}, v_{*}^{\prime}\right) d v d v_{*} d \theta d x d s \\
& +c \int_{t}^{t+t_{0}} \int_{\left|v-v_{*}\right|<\lambda} B \chi_{j} f^{\sharp}(s, x, v) f\left(s, x+s v_{1}, v_{*}\right) d v d v_{*} d \theta d x d s \\
& \leq \int_{\left|x-x_{0}\right|<\delta_{2}} f^{\sharp}\left(t+t_{0}, x, v\right) d x d v+\frac{C_{1}+C_{2} T}{\lambda^{2}}+c t_{0} \lambda^{2} \int f^{\sharp}(t, x, v) d x d v \\
& \leq \frac{1}{\Lambda^{2}} \int v^{2} f_{0} d x d v+c \delta_{2} \Lambda^{2}+\frac{C_{1}+C_{2} T}{\lambda^{2}}+c t_{0} \lambda^{2} \int f_{0}(x, v) d x d v
\end{aligned}
$$

Depending on $\delta_{1}$, suitably choosing $\Lambda$ and then $\delta_{2}, \lambda$ and then $t_{0}$, the lemma follows.

The previous lemmas imply a $t$-dependent bound for the $v$-integral of $f_{j}^{\#}$ only depending on $\int f_{0}(x, v) d x d v$ and on $\int|v|^{2} f_{0}(x, v) d x d v$, as will now be proved.

\section{Lemma 3.5}

Given $T>0$, the solution $f_{j}$ of (3.1) satisfies

$$
\int \sup _{(t, x) \in[0, T] \times[0,1]} f_{j}^{\sharp}(t, x, v) d v \leq c_{1}(T), \quad j \in \mathbb{N}^{*},
$$

where $c_{1}(T)$ only depends on $T, \int f_{0}(x, v) d x d v$ and $\int|v|^{2} f_{0}(x, v) d x d v$.

\section{Proof of Lemma 3.5.}

For any $a, b \in \mathbb{R}$, denote by $I(a, b)$ the interval with end points $a$ and $b$.

Denote by $E(x)$ the integer part of $x \in \mathbb{R}, E(x) \leq x<E(x)+1$.

As in the proof of Lemma 3.3,

$$
\begin{aligned}
\sup _{s \leq t} f^{\sharp}(s, x, v) & \leq f_{0}(x, v)+\int_{0}^{t} Q_{j}^{+}(f)\left(s, x+s v_{1}, v\right) d s=f_{0}(x, v) \\
+\int_{0}^{t} & \int B \chi_{j} f\left(s, x+s v_{1}, v^{\prime}\right) f\left(s, x+s v_{1}, v_{*}^{\prime}\right) F_{j}(f)\left(s, x+s v_{1}, v\right) F_{j}(f)\left(s, x+s v_{1}, v_{*}\right) d v_{*} d \omega d s \\
& \leq f_{0}(x, v)+c A,
\end{aligned}
$$

where

$$
A=\int_{0}^{t} \int B \chi_{j} \sup _{\tau \in[0, t]} f^{\#}\left(\tau, x+s\left(v_{1}-v_{1}^{\prime}\right), v^{\prime}\right) \sup _{\tau \in[0, t]} f^{\#}\left(\tau, x+s\left(v_{1}-v_{* 1}^{\prime}\right), v_{*}^{\prime}\right) d v_{*} d \omega d s .
$$

For $\theta$ outside of the angular cutoff (2.2), let $n$ be the unit vector in the direction $v-v^{\prime}$, and $n_{\perp}$ the orthogonal unit vector in the direction $v-v_{*}^{\prime}$. With $e_{1}$ a unit vector in the $x$-direction,

$$
\max \left(\left|n \cdot e_{1}\right|,\left|n_{\perp} \cdot e_{1}\right|\right) \geq \frac{1}{\sqrt{2}}
$$


For $\delta_{2}>0$ that will be fixed later, split $A$ into $A_{1}+A_{2}+A_{3}+A_{4}$, where

$$
\begin{aligned}
& A_{1}=\int_{0}^{t} \int_{\left|n \cdot e_{1}\right| \geq \frac{1}{\sqrt{2}}, t\left|v_{1}-v_{1}^{\prime}\right|>\delta_{2}} B \chi_{j} \sup _{\tau \in[0, t]} f^{\#}\left(\tau, x+s\left(v_{1}-v_{1}^{\prime}\right), v^{\prime}\right) \sup _{\tau \in[0, t]} f^{\#}\left(\tau, x+s\left(v_{1}-v_{* 1}^{\prime}\right), v_{*}^{\prime}\right) d v_{*} d \omega d s, \\
& A_{2}=\int_{0}^{t} \int_{\left|n \cdot e_{1}\right| \geq \frac{1}{\sqrt{2}}, t\left|v_{1}-v_{1}^{\prime}\right|<\delta_{2}} B \chi_{j} \sup _{\tau \in[0, t]} f^{\#}\left(\tau, x+s\left(v_{1}-v_{1}^{\prime}\right), v^{\prime}\right) \sup _{\tau \in[0, t]} f^{\#}\left(\tau, x+s\left(v_{1}-v_{* 1}^{\prime}\right), v_{*}^{\prime}\right) d v_{*} d \omega d s, \\
& A_{3}= \int_{0}^{t} \int_{\left|n_{\perp} \cdot e_{1}\right| \geq \frac{1}{\sqrt{2}}, t\left|v_{1}-v_{1}^{\prime}\right|>\delta_{2}} B \chi_{j} \sup _{\tau \in[0, t]} f^{\#}\left(\tau, x+s\left(v_{1}-v_{1}^{\prime}\right), v^{\prime}\right) \sup _{\tau \in[0, t]} f^{\#}\left(\tau, x+s\left(v_{1}-v_{* 1}^{\prime}\right), v_{*}^{\prime}\right) d v_{*} d \omega d s, \\
& A_{4}=\int_{0}^{t} \int_{\left|n_{\perp} \cdot e_{1}\right| \geq \frac{1}{\sqrt{2}}, t\left|v_{1}-v_{1}^{\prime}\right|<\delta_{2}} B \chi_{j} \sup _{\tau \in[0, t]} f^{\#}\left(\tau, x+s\left(v_{1}-v_{1}^{\prime}\right), v^{\prime}\right) \sup _{\tau \in[0, t]} f^{\#}\left(\tau, x+s\left(v_{1}-v_{* 1}^{\prime}\right), v_{*}^{\prime}\right) d v_{*} d \omega d s .
\end{aligned}
$$

In $A_{1}$ and $A_{2}$, bound the factor $\sup _{\tau \in[0, t]} f^{\sharp}\left(\tau, x+s\left(v_{1}-v_{* 1}^{\prime}\right), v_{*}^{\prime}\right)$ by its supremum over $x \in[0,1]$, and make the change of variables

$$
s \rightarrow y=x+s\left(v_{1}-v_{1}^{\prime}\right)
$$

with Jacobian

$$
\frac{D s}{D y}=\frac{1}{\left|v_{1}-v_{1}^{\prime}\right|}=\frac{1}{\left|v-v_{*}\right|\left|\left(n, \frac{v-v_{*}}{\mid v-v_{*}}\right)\right|\left|n_{1}\right|} \leq \frac{\sqrt{2}}{\gamma \gamma^{\prime}}
$$

It holds that

$$
A_{1} \leq \int_{t\left|v_{1}-v_{1}^{\prime}\right|>\delta_{2}} \frac{B \chi_{j}}{\left|v_{1}-v_{1}^{\prime}\right|}\left(\int_{y \in I\left(x, x+t\left(v_{1}-v_{1}^{\prime}\right)\right.} \sup _{\tau \in[0, t]} f^{\#}\left(\tau, y, v^{\prime}\right) d y\right) \sup _{(\tau, X) \in[0, t] \times[0,1]} f^{\#}\left(\tau, X, v_{*}^{\prime}\right) d v_{*} d \omega,
$$

and

$$
A_{2} \leq \frac{\sqrt{2}}{\gamma \gamma^{\prime}} \int_{\left|n \cdot e_{1}\right| \geq \frac{1}{\sqrt{2}}, t\left|v_{1}-v_{1}^{\prime}\right|<\delta_{2}} B \chi_{j}\left(\int_{|y-x|<\delta_{2}} \sup _{\tau \in[0, t]} f^{\#}\left(\tau, y, v^{\prime}\right) d y\right) \sup _{(\tau, X) \in[0, t] \times[0,1]} f^{\#}\left(\tau, X, v_{*}^{\prime}\right) d v_{*} d \omega .
$$

Then, performing the change of variables $\left(v, v_{*}, \omega\right) \rightarrow\left(v^{\prime}, v_{*}^{\prime},-\omega\right)$,

$$
\begin{aligned}
& \int \sup _{x \in[0,1]} A_{1} d v \\
& \leq \int_{t\left|v_{1}-v_{1}^{\prime}\right|>\delta_{2}} \frac{B \chi_{j}}{\left|v_{1}-v_{1}^{\prime}\right|} \sup _{x \in[0,1]}\left(\int_{y \in I\left(x, x+t\left(v_{1}^{\prime}-v_{1}\right)\right.} \sup _{\tau \in[0, t]} f^{\#}(\tau, y, v) d y\right) \sup _{(\tau, X) \in[0, t] \times[0,1]} f^{\#}\left(\tau, X, v_{*}\right) d v d v_{*} d \omega,
\end{aligned}
$$

so that

$$
\begin{aligned}
& \int \sup _{x \in[0,1]} A_{1} d v \\
& \leq \int_{t\left|v_{1}-v_{1}^{\prime}\right|>\delta_{2}} \frac{B \chi_{j}}{\left|v_{1}-v_{1}^{\prime}\right|} \sup _{x \in[0,1]}\left(\int_{y \in I\left(x, x+E\left(t\left(v_{1}^{\prime}-v_{1}\right)+1\right)\right.} \sup _{\tau \in[0, t]} f^{\#}(\tau, y, v) d y\right) \sup _{(\tau, X) \in[0, t] \times[0,1]} f^{\#}\left(\tau, X, v_{*}\right) d v d v_{*} d \omega \\
& =\int_{t\left|v_{1}-v_{1}^{\prime}\right|>\delta_{2}} \frac{B \chi_{j}}{\left|v_{1}-v_{1}^{\prime}\right|}\left|E\left(t\left(v_{1}^{\prime}-v_{1}\right)+1\right)\right|\left(\int_{0}^{1} \sup _{\tau \in[0, t]} f^{\#}(\tau, y, v) d y\right) \sup _{(\tau, X) \in[0, t] \times[0,1]} f^{\#}\left(\tau, X, v_{*}\right) d v d v_{*} d \omega \\
& \leq t\left(1+\frac{1}{\delta_{2}}\right) \int B \chi_{j}\left(\int_{0}^{1} \sup _{\tau \in[0, t]} f^{\#}(\tau, y, v) d y\right) \sup _{(\tau, X) \in[0, t] \times[0,1]} f^{\#}\left(\tau, X, v_{*}\right) d v d v_{*} d \omega \\
& \leq B_{0} \pi t\left(1+\frac{1}{\delta_{2}}\right) \int \sup _{\tau \in[0, t]} f^{\#}(\tau, y, v) d y d v \int_{(\tau, X) \in[0, t] \times[0,1]} f^{\#}\left(\tau, X, v_{*}\right) d v_{*} .
\end{aligned}
$$


Apply Lemma 3.3, so that

$$
\int \sup _{x \in[0,1]} A_{1} d v \leq\left(c_{1}^{\prime}+c_{2}^{\prime} T\right) B_{0} \pi t\left(1+\frac{1}{\delta_{2}}\right) \int \sup _{(\tau, X) \in[0, t] \times[0,1]} f^{\#}\left(\tau, X, v_{*}\right) d v_{*} .
$$

Moreover, performing the change of variables $\left(v, v_{*}, \omega\right) \rightarrow\left(v_{*}^{\prime}, v^{\prime},-\omega\right)$,

$$
\int \sup _{x \in[0,1]} A_{2} d v \leq \frac{B_{0} \pi \sqrt{2}}{\gamma \gamma^{\prime}} \sup _{x \in[0,1]}\left(\int_{|y-x|<\delta_{2}} \sup _{\tau \in[0, t]} f^{\#}\left(\tau, y, v_{*}\right) d y d v_{*}\right) \int \sup _{(\tau, X) \in[0, t] \times[0,1]} f^{\#}(\tau, X, v) d v .
$$

Given $\delta_{1}=\frac{\gamma \gamma^{\prime}}{4 B_{0} \pi \sqrt{2}}$, apply Lemma 3.4 with the corresponding $\delta_{2}$ and $t_{0}$, so that for $t \leq t_{0}$,

$$
\int \sup _{x \in[0,1]} A_{2} d v \leq \frac{1}{4} \int \sup _{(\tau, X) \in[0, t] \times[0,1]} f^{\#}(\tau, X, v) d v .
$$

The terms $A_{3}$ and $A_{4}$ are treated similarly, with the change of variables $s \rightarrow y=x+s\left(v_{1}-v_{* 1}^{\prime}\right)$. Using (3.9)-(3.10) and the corresponding bounds obtained for $A_{3}$ and $A_{4}$ leads to

$$
\begin{gathered}
\int \sup _{(s, x) \in[0, t] \times[0,1]} f^{\#}(s, x, v) d v \leq 2 \int \sup _{x \in[0,1]} f_{0}(x, v) d v \\
+4\left(c_{1}^{\prime}+c_{2}^{\prime} T\right) B_{0} \pi t\left(1+\frac{1}{\delta_{2}}\right) \int \sup _{(s, x) \in[0, t] \times[0,1]} f^{\#}(s, x, v) d v, \quad t \leq t_{0} .
\end{gathered}
$$

Hence for $t \leq \min \left(t_{0},\left(8\left(c_{1}^{\prime}+c_{2}^{\prime} T\right) B_{0} \pi^{2}\left(1+\frac{1}{\delta_{2}}\right)\right)^{-1}\right.$

$$
\int \sup _{(s, x) \in[0, t] \times[0,1]} f^{\#}(s, x, v) d v \leq 4 \int \sup _{x \in[0,1]} f_{0}(x, v) d v .
$$

Since $c_{1}^{\prime}, c_{2}^{\prime}$, and $t_{0}$ only depend on $\int\left(1+|v|^{2}\right) f_{0}(x, v) d x d v$ and $T$, it follows that the argument can be repeated up to $t=T$. This completes the proof of the lemma.

\section{Proof of the main theorem and the asymptotic behavior.}

The following two preliminary lemmas are needed for the control of large velocities.

\section{Lemma 4.1}

Given $t>0$, there is a constant $c_{t}>0$ such that the solutions of (3.1) satisfy

$$
\sup _{j \in \mathbb{N}^{*}} \int_{0}^{1} \int_{|v|>\lambda}|v| \sup _{s \leq t} f_{j}^{\sharp}(s, x, v) d v d x \leq \frac{c_{t}}{\lambda} .
$$

Proof of Lemma 4.1.

For convenience $j$ is dropped from the notation $f_{j}$. As in Section 3 ,

$$
\sup _{s \leq t} f^{\sharp}(s, x, v) \leq f_{0}(x, v)+\int_{0}^{t} Q_{j}^{+}(f)\left(s, x+s v_{1}, v\right) d s .
$$


Integration with respect to $(x, v)$ for $|v|>\lambda$, gives

$$
\begin{array}{r}
\int_{0}^{1} \int_{|v|>\lambda}|v| \sup _{s \leq t} f^{\sharp}(s, x, v) d v d x \leq \iint_{|v|>\lambda}|v| f_{0}(x, v) d v d x+\int_{0}^{t} \int_{|v|>\lambda} B \chi_{j} \\
|v| f\left(s, x+s v_{1}, v^{\prime}\right) f\left(s, x+s v_{1}, v_{*}^{\prime}\right) F(f)\left(s, x+s v_{1}, v\right) F(f)\left(s, x+s v_{1}, v_{*}\right) d v d v_{*} d \omega d x d s .
\end{array}
$$

Here in the last integral, either $\left|v^{\prime}\right|$ or $\left|v_{*}^{\prime}\right|$ is the largest and larger than $\frac{\lambda}{\sqrt{2}}$. The two cases are symmetric, and we discuss the case $\left|v^{\prime}\right| \geq\left|v_{*}^{\prime}\right|$. After a translation in $x$, the integrand is estimated from above by $c\left|v^{\prime}\right| f^{\#}\left(s, x, v^{\prime}\right) \sup _{x \in[0,1], s \leq t} f^{\#}\left(s, x, v_{*}^{\prime}\right)$. The change of variables $\left(v, v_{*}, \omega\right) \rightarrow\left(v^{\prime}, v_{*}^{\prime},-\omega\right)$, the integration over $\left(s, x, v, v_{*}, \omega\right) \in[0, t] \times[0,1] \times\left\{v \in \mathbb{R}^{2} ;|v|>\frac{\lambda}{\sqrt{2}}\right\} \times \mathbb{R}^{2} \times S^{1}$ and Lemma 3.5 give the bound

$$
\frac{c}{\lambda}\left(\int_{0}^{t} \int|v|^{2} f^{\#}(s, x, v) d x d v d s\right)\left(\int \sup _{s \leq t, x \in[0,1]} f^{\#}\left(s, x, v_{*}\right) d v_{*}\right) \leq \frac{c t c_{1}(t)}{\lambda} \int|v|^{2} f_{0}(x, v) d x d v .
$$

The lemma follows.

\section{Lemma 4.2}

Given $t>0$ and $\lambda>2$, there is a constant $c_{t}^{\prime}>0$, such that the solutions $f_{j}$ of (3.1) satisfy

$$
\sup _{j \in \mathbb{N}^{*}} \int_{|v|>\lambda} \sup _{(s, x) \in[0, t] \times[0,1]} f_{j}^{\sharp}(s, x, v) d v \leq \frac{c_{t}^{\prime}}{\sqrt{\lambda}} .
$$

Proof of Lemma 4.2 .

Take $\lambda>2$. As above,

$$
\int_{|v|>\lambda} \sup _{(s, x) \in[0, t] \times[0,1]} f^{\sharp}(s, x, v) d v \leq \int_{|v|>\lambda} \sup _{x \in[0,1]} f_{0}(x, v) d v+C,
$$

where

$$
C=c \int_{|v|>\lambda} \sup _{x \in[0,1]} \int_{0}^{t} \int B \chi_{j} f^{\#}\left(s, x+s\left(v_{1}-v_{1}^{\prime}\right), v^{\prime}\right) f^{\#}\left(s, x+s\left(v_{1}-v_{* 1}^{\prime}\right), v_{*}^{\prime}\right) d v d v_{*} d \omega d s .
$$

For $v^{\prime}, v_{*}^{\prime}$ outside of the angular cutoff (2.2), let $n$ be the unit vector in the direction $v-v^{\prime}$, and $n_{\perp}$ the orthogonal unit vector in the direction $v-v_{*}^{\prime}$. Let $e_{1}$ be a unit vector in the $x$-direction. Split $C$ as $C=\sum_{1 \leq i \leq 6} C_{i}$, where $C_{1}$ (resp. $C_{2}, C_{3}$ ) refers to integration on

$$
\left\{\left(v_{*}, \omega\right) ; \quad n \cdot e_{1} \geq \frac{1}{\sqrt{2}}, \quad\left|v^{\prime}\right| \geq\left|v_{*}^{\prime}\right|\right\},
$$

$$
\left(\operatorname{resp} .\left\{\left(v_{*}, \omega\right) ; n \cdot e_{1} \geq \sqrt{1-\frac{1}{\lambda}},\left|v^{\prime}\right| \leq\left|v_{*}^{\prime}\right|\right\}, \quad\left\{\left(v_{*}, \omega\right) ; n \cdot e_{1} \in\left[\frac{1}{\sqrt{2}}, \sqrt{1-\frac{1}{\lambda}}\right],\left|v^{\prime}\right| \leq\left|v_{*}^{\prime}\right|\right\}\right),
$$

and analogously for $C_{i}, 4 \leq i \leq 6$, with $n$ replaced by $n_{\perp}$. By symmetry, $C_{i}, 4 \leq i \leq 6$ can be treated as $C_{i}, 1 \leq i \leq 3$, so we only discuss the control of $C_{i}, 1 \leq i \leq 3$.

By the change of variables $\left(v, v_{*}, \omega\right) \rightarrow\left(v^{\prime}, v_{*}^{\prime},-\omega\right)$, and noticing that $\left|v^{\prime}\right| \geq \frac{\lambda}{\sqrt{2}}$ in the domain of integration of $C_{1}$, it holds that

$$
\begin{aligned}
C_{1} & \leq \int_{|v|>\frac{\lambda}{\sqrt{2}}} \sup _{x \in[0,1]} \int_{0}^{t} \int_{n \cdot e_{1} \geq \frac{1}{\sqrt{2}}} B \chi_{j} f^{\#}\left(s, x+s\left(v_{1}^{\prime}-v_{1}\right), v\right) f^{\#}\left(s, x+s\left(v_{1}^{\prime}-v_{* 1}\right), v_{*}\right) d v_{*} d \omega d s d v \\
& \leq \int_{|v|>\frac{\lambda}{\sqrt{2}}} \sup _{x \in[0,1]} \int_{0}^{t} \int_{n \cdot e_{1} \geq \frac{1}{\sqrt{2}}} B \chi_{j} \sup _{\tau \in[0, t]} f^{\#}\left(\tau, x+s\left(v_{1}^{\prime}-v_{1}\right), v\right) \sup _{(\tau, X) \in[0, t] \times[0,1]} f^{\#}\left(\tau, X, v_{*}\right) d v_{*} d \omega d s d v .
\end{aligned}
$$


With the change of variables $s \rightarrow y=x+s\left(v_{1}^{\prime}-v_{1}\right)$,

$$
\begin{aligned}
C_{1} & \leq \int_{|v|>\frac{\lambda}{\sqrt{2}}} \sup _{x \in[0,1]} \int_{n \cdot e_{1} \geq \frac{1}{\sqrt{2}}} \int_{y \in I\left(x, x+t\left(v_{1}^{\prime}-v_{1}\right)\right)} \frac{B \chi_{j}}{\left|v_{1}^{\prime}-v_{1}\right|} \sup _{\tau \in[0, t]} f^{\#}(\tau, y, v) \sup _{(\tau, X) \in[0, t] \times[0,1]} f^{\#}\left(\tau, X, v_{*}\right) d y d v_{*} d \omega d v \\
& \leq \int_{|v|>\frac{\lambda}{\sqrt{2}}} \int_{n \cdot e_{1} \geq \frac{1}{\sqrt{2}}} \frac{\left.\mid E\left(t\left(v_{1}^{\prime}-v_{1}\right)\right)+1\right) \mid}{\left|v_{1}^{\prime}-v_{1}\right|} \int_{0}^{1} B \chi_{j} \sup _{\tau \in[0, t]} f^{\#}(\tau, y, v) \sup _{(\tau, X) \in[0, t] \times[0,1]} f^{\#}\left(\tau, X, v_{*}\right) d y d v_{*} d \omega d v .
\end{aligned}
$$

Moreover,

$$
\left.\mid E\left(t\left(v_{1}^{\prime}-v_{1}\right)\right)+1\right)|\leq t| v_{1}^{\prime}-v_{1}\left|+1 \leq\left(t+\frac{\sqrt{2}}{\gamma \gamma^{\prime}}\right)\right| v_{1}^{\prime}-v_{1} \mid .
$$

Consequently,

$$
\begin{aligned}
C_{1} & \leq c(t+1) \int_{0}^{1} \int_{|v|>\frac{\lambda}{\sqrt{2}}} \sup _{\tau \in[0, t]} f^{\#}(\tau, y, v) d y d v \int \sup _{(\tau, X) \in[0, t] \times[0,1]} f^{\#}\left(\tau, X, v_{*}\right) d v_{*} \\
& \leq \frac{c(t+1)}{\lambda} \int_{0}^{1} \int_{|v|>\frac{\lambda}{\sqrt{2}}}|v| \sup _{\tau \in[0, t]} f^{\#}(\tau, y, v) d y d v \int \sup _{(\tau, X) \in[0, t] \times[0,1]} f^{\#}\left(\tau, X, v_{*}\right) d v_{*} .
\end{aligned}
$$

By Lemma 3.5 and Lemma 4.1,

$$
C_{1} \leq \frac{c}{\lambda^{2}}(t+1) c_{t} c_{1}(t)
$$

Moreover,

$$
\begin{aligned}
C_{2} & \leq \int_{\left|v^{\prime}\right|>\lambda,\left|v_{*}\right|>|v|, n \cdot e_{1} \geq \sqrt{1-\frac{1}{\lambda}}} \frac{B \chi_{j}}{\left|v_{1}^{\prime}-v_{1}\right|} \\
& \sup _{x \in[0,1]} \int_{y \in I\left(x, x+t\left(v_{1}^{\prime}-v_{1}\right)\right.} \sup _{\tau \in[0, t]} f^{\#}(\tau, y, v) \sup _{(\tau, X) \in[0, t] \times[0,1]} f^{\#}\left(\tau, X, v_{*}\right) d y d v d v_{*} d \omega \\
& \leq c(t+1) \int_{n \cdot e_{1} \geq \sqrt{1-\frac{1}{\lambda}}} d \omega \int_{\tau \in[0, t]} f^{\#}(\tau, y, v) d y d v \int_{(\tau, X) \in[0, t] \times[0,1]} \sup _{\tau}\left(\tau, X, v_{*}\right) d v_{*} \\
& \leq \frac{c}{\sqrt{\lambda}}(t+1)^{2} c_{1}(t),
\end{aligned}
$$

by Lemma 3.3 and Lemma 3.5. Finally,

$$
\begin{aligned}
C_{3} \leq & \int_{\left|v_{*}\right|>\frac{\lambda}{\sqrt{2}}, \frac{1}{\sqrt{\lambda}} \leq n_{\perp} \cdot e_{1} \leq \frac{1}{\sqrt{2}}} \sup _{(\tau, X) \in[0, t] \times[0,1]} f^{\#}(\tau, X, v) \frac{B \chi_{j}}{\left|v_{1}^{\prime}-v_{* 1}\right|} \\
& \sup _{x \in[0,1]}\left(\int_{y \in I\left(x, x+t\left(v_{1}^{\prime}-v_{* 1}\right)\right.} \sup _{\tau \in[0, t]} f^{\#}\left(\tau, y, v_{*}\right) d y\right) d v d v_{*} d \omega \\
& \leq c(t+1) \sqrt{\lambda}\left(\int_{(\tau, X) \in[0, t] \times[0,1]} f^{\#}(\tau, X, v) d v\right)\left(\int_{\left|v_{*}\right|>\frac{\lambda}{\sqrt{2}}} \sup _{\tau \in[0, t]} f^{\#}\left(\tau, y, v_{*}\right) d y d v_{*}\right) .
\end{aligned}
$$

By Lemma 3.5,

$$
C_{3} \leq c(t+1) \sqrt{\lambda} c_{1}(t) \int_{\left|v_{*}\right|>\frac{\lambda}{\sqrt{2}}} \sup _{\tau \in[0, t]} f^{\#}\left(\tau, y, v_{*}\right) d y d v_{*},
$$


and so by Lemma 4.1,

$$
C_{3} \leq \frac{c}{\sqrt{\lambda}}(t+1) c_{1}(t) c_{t}
$$

The lemma follows.

Using the previous lemmas, the results in Section 3, and an initial layer analysis, the main result of the paper follows.

Proof of Theorem 2.1.

For any $T>0$, we shall prove the convergence in $C\left([0, T] ; L^{1}\left([0, T] \times \mathbb{R}^{2}\right)\right)$ of the sequence $\left(f_{j}\right)$ to a solution $f$ of (2.5). Denote by

$$
\tilde{\nu}_{j}(f):=\int B \chi_{j} f^{\prime} f_{*}^{\prime} F_{j}\left(f_{*}\right) d v_{*} d \omega, \quad \nu_{j}(f):=\int B \chi_{j} f_{*} F_{j}\left(f^{\prime}\right) F_{j}\left(f_{*}^{\prime}\right) d v_{*} d \omega,
$$

so that

$$
Q_{j}(f)=F_{j}(f) \tilde{\nu}_{j}(f)-f \nu_{j}(f) .
$$

Consider

$$
\nu_{j}\left(f_{j}\right)^{\sharp}(t, x, v)=\int B \chi_{j} f_{j}\left(t, x+t v_{1}, v_{*}\right) F_{j}\left(f_{j}\left(t, x+t v_{1}, v^{\prime}\right)\right) F_{j}\left(f_{j}\left(t, x+t v_{1}, v_{*}^{\prime}\right)\right) d v_{*} d \omega .
$$

With the angular cut-off $(2.2), v_{*} \rightarrow v^{\prime}$ and $v_{*} \rightarrow v_{*}^{\prime}$ are changes of variables. Indeed, if the polar coordinates of $v_{*}-v$ are $\left(r_{*}, \varphi\right)$ and $\theta$ is the angle between $v_{*}-v$ and $n$, then the polar coordinates of $v^{\prime}-v\left(\right.$ resp. $\left.v_{*}^{\prime}-v\right)$ are $\left(\left|r_{*} \cos \theta\right|, \varphi+\theta\right)\left(\right.$ resp. $\left.\left(\left|r_{*} \sin \theta\right|, \varphi+\theta+\frac{\pi}{2}\right)\right)$. It follows from the angular cut-off (2.2), that the Jacobians $\frac{D v_{*}}{D v^{\prime}}=\frac{1}{|\cos \theta|}$ (resp. $\frac{D v_{*}}{D v_{*}^{\prime}}=\frac{1}{|\sin \theta|}$ are bounded. Using these changes of variables and Lemma 3.5, for $\omega$ outside the integration cut-off, the measure of the set

$$
Z_{(j, t, x, v, \omega)}:=\left\{v_{*} ; f\left(t, x+t v_{1}, v^{\prime}\right)>\frac{1}{2} \quad \text { or } \quad f\left(t, x+t v_{1}, v_{*}^{\prime}\right)>\frac{1}{2}\right\}
$$

is uniformly bounded with respect to $(x, v, \omega), t \leq T$, and $j \in \mathbb{N}^{*}$. Take $j_{T}$ so large that $\pi j_{T}^{2}$ is at least eight times this uniform bound. Notice that here $j_{T}$ only depends on $T$ and $\int\left(1+v^{2}\right) f_{0}(x, v) d x d v$. Using the exponential form for the solution, one gets using Lemma 3.5 that

$$
f_{j}^{\sharp}\left(t, x, v_{*}\right) \geq c_{1 T} f_{0}\left(x, v_{*}\right)>0, \quad j \geq j_{T}, \quad t \leq T,
$$

with $c_{1 T}$ independent of $j \geq j_{T}$. It follows from (4.3) and the third assumption in (2.4) that

$$
\nu_{j}\left(f_{j}\right)^{\sharp}(t, x, v)>c_{2 T}>0, \quad(t, x, v) \in[0, T] \times[0,1] \times\left\{v \in \mathbb{R}^{2} ;|v| \leq j\right\},
$$

uniformly with respect to $j \geq j_{T}$, and with $c_{2 T}$ only depending on $T$ and $f_{0}$. Using again the $v_{*} \rightarrow v^{\prime}$ change of variables together with Lemma 3.5, one obtains that for some constant $c_{3 T}>0$

$$
\tilde{\nu}_{j}^{\sharp}\left(f_{j}\right)(t, x, v) \leq c_{3 T}, \quad j \geq j_{T}, \quad(t, x, v) \in[0, T] \times[0,1] \times\left\{v \in \mathbb{R}^{2} ;|v| \leq j\right\} .
$$


The functions defined on $\left.] 0, \frac{1}{\alpha}\right]$ by $x \rightarrow \frac{F_{j}(x)}{x}$ are uniformly bounded from above with respect to $j$ by

$$
x \rightarrow c \alpha^{\alpha-1} \frac{(1-\alpha x)^{\alpha}}{x},
$$

that is continuous and decreasing to zero at $x=\frac{1}{\alpha}$. Hence there is $\left.\mu \in\right] 0, \frac{1}{\alpha}[$ such that

$$
x \in\left[\frac{1}{\alpha}-\mu, \frac{1}{\alpha}\right] \quad \text { implies } \quad \frac{F_{j}(x)}{x} \leq \frac{c_{2 T}}{4 c_{3 T}}, \quad j \geq j_{T} .
$$

Consequently, for $j \geq j_{T}$,

$$
\begin{aligned}
f_{j}^{\sharp}(t, x, v) \in\left[\frac{1}{\alpha}-\mu, \frac{1}{\alpha}\right] \Rightarrow D_{t} f_{j}^{\sharp}(t, x, v) & =\left(F_{j}\left(f_{j}^{\sharp}\right) \tilde{\nu}_{j}^{\sharp}-\frac{1}{2} f_{j}^{\sharp} \nu_{j}^{\sharp}\right)(t, x, v)-\frac{1}{2} f_{j}^{\sharp} \nu_{j}^{\sharp}(t, x, v) \\
& <-\frac{1}{2} f_{j}^{\sharp} \nu_{j}^{\sharp}(t, x, v) \\
& <-\frac{1}{2}\left(\frac{1}{\alpha}-\mu\right) c_{2 T}:=-b_{1} .
\end{aligned}
$$

This gives a maximum time $t_{1}=\frac{\mu}{b_{1}}$ for $f_{j}^{\#}$ to reach $\frac{1}{\alpha}-\mu$ from an initial value $\left.\left.f_{0}(x, v) \in\right] \frac{1}{\alpha}-\mu, \frac{1}{\alpha}\right]$. On this time interval $D_{t} f_{j}^{\sharp} \leq-b_{1}$. If $t_{1} \geq T$, then at $t=T$ the value of $f_{j}^{\#}$ is bounded from above by $\frac{1}{\alpha}-b_{1} T:=\frac{1}{\alpha}-\mu^{\prime}$ with $0<\mu^{\prime} \leq \mu$. Take $t_{m}=\min \left(t_{1}, T\right)$, and from now on $\mu=t_{m} b_{1}$. For any $(x, v)$, if $f_{j}(0, x, v)<\frac{1}{\alpha}-\mu$ were to reach $\frac{1}{\alpha}-\mu$ at $(t, x, v)$ with $t \leq t_{m}$, then $D_{t} f_{j}^{\#}(t, x, v) \leq-b_{1}$, which excludes such a possibility. It follows that $f_{j} \leq \frac{1}{\alpha}-\mu$ everywhere for $t \in\left[t_{m}, T\right]$, and that

$$
f_{j}^{\sharp}(t, x, v) \leq \frac{1}{\alpha}-b_{1} t .
$$

for $t \in\left[0, t_{m}\right]$. The previous estimates leading to the definition of $t_{m}$ are independent of $j \geq j_{T}$.

Let us prove that $\left(f_{j}\right)$ converges in $L^{1}\left([0, T] \times[0,1] \times \mathbb{R}^{2}\right)$ when $j \rightarrow \infty$.

We shall prove that given $\beta>0$, there exists $a \geq \max \left\{1, j_{T}\right\}$, so that

$$
\sup _{t \in[0, T]} \int\left|g_{j}(t, x, v)\right| d x d v<\beta, \quad j>a
$$

where $g_{j}=f_{j}-f_{a}$. The function $g_{j}$ satisfies the equation

$$
\begin{aligned}
\partial_{t} g_{j}+p_{1} \partial_{x} g_{j} & =\int\left(\chi_{j}-\chi_{a}\right) B\left(f_{j}^{\prime} f_{j *}^{\prime} F_{j}\left(f_{j}\right) F_{j}\left(f_{j *}\right)-f_{j} f_{j *} F_{j}\left(f_{j}^{\prime}\right) F_{j}\left(f_{j *}^{\prime}\right)\right) d v_{*} d \omega \\
& +\int \chi_{a} B\left(f_{j}^{\prime} f_{j *}^{\prime}-f_{a}^{\prime} f_{a *}^{\prime}\right) F_{j}\left(f_{j}\right) F_{j}\left(f_{j *}\right) d v_{*} d \omega \\
& -\int \chi_{a} B\left(f_{j} f_{j *}-f_{a} f_{a *}\right) F_{j}\left(f_{j}^{\prime}\right) F_{j}\left(f_{j *}^{\prime}\right) d v_{*} d \omega \\
& +\int \chi_{a} B f_{a}^{\prime} f_{a *}^{\prime}\left(F_{j}\left(f_{j *}\right)\left(F_{j}\left(f_{j}\right)-F_{j}\left(f_{a}\right)\right)+F_{a}\left(f_{a}\right)\left(F_{j}\left(f_{j *}\right)-F_{j}\left(f_{a *}\right)\right)\right) d v_{*} d \omega \\
& +\int \chi_{a} B f_{a}^{\prime} f_{a *}^{\prime}\left(F_{j}\left(f_{j *}\right)\left(F_{j}\left(f_{a}\right)-F_{a}\left(f_{a}\right)\right)+F_{a}\left(f_{a}\right)\left(F_{j}\left(f_{a *}\right)-F_{a}\left(f_{a *}\right)\right)\right) d v_{*} d \omega \\
& -\int \chi_{a} B f_{a} f_{a *}\left(F_{j}\left(f_{j *}^{\prime}\right)\left(F_{j}\left(f_{j}^{\prime}\right)-F_{j}\left(f_{a}^{\prime}\right)\right)+F_{a}\left(f_{a}^{\prime}\right)\left(F_{j}\left(f_{j *}^{\prime}\right)-F_{j}\left(f_{a *}^{\prime}\right)\right)\right) d v_{*} d \omega \\
& -\int \chi_{a} B f_{a} f_{a *}\left(F_{j}\left(f_{j *}^{\prime}\right)\left(F_{j}\left(f_{a}^{\prime}\right)-F_{a}\left(f_{a}^{\prime}\right)\right)+F_{a}\left(f_{a}^{\prime}\right)\left(F_{j}\left(f_{a *}^{\prime}\right)-F_{a}\left(f_{a *}^{\prime}\right)\right)\right) d v_{*} d \omega .
\end{aligned}
$$


Moreover, using Lemma 3.5

$$
\begin{gathered}
\int\left(\chi_{j}-\chi_{a}\right) B\left(f_{j}^{\prime} f_{j *}^{\prime} F_{j}\left(f_{j}\right) F_{j}\left(f_{j *}\right)+f_{j} f_{j *} F_{j}\left(f_{j}^{\prime}\right) F_{j}\left(f_{j *}^{\prime}\right)\right) d x d v d v_{*} d \omega \\
\leq c \int_{|v|>\frac{a}{\sqrt{2}}} f_{j}(t, x, v) d x d v \\
\leq \frac{c}{a^{2}} \text { by the conservation of energy of } f_{j}, \\
\int \chi_{a} B\left|f_{j} f_{j *}-f_{a} f_{a *}\right| F_{j}\left(f_{j}^{\prime}\right) F_{j}\left(f_{j *}^{\prime}\right) d x d v d v_{*} d \omega \\
\leq c\left(\int_{(t, x) \in[0, T] \times[0,1]} f_{j}^{\sharp}(t, x, v) d v+\int_{(t, x) \in[0, T] \times[0,1]} f_{a}^{\sharp}(t, x, v) d v\right) \int\left|\left(f_{j}^{\sharp}-f_{a}^{\sharp}\right)(t, x, v)\right| d x d v \\
\leq c \int\left|\left(f_{j}^{\sharp}-f_{a}^{\sharp}\right)(t, x, v)\right| d x d v \quad \text { by Lemma } 3.5 .
\end{gathered}
$$

Next,

$$
\begin{aligned}
& \int \chi_{a} B\left(f_{a}^{\prime} f_{a *}^{\prime} F_{j}\left(f_{j *}\right)\left|F_{j}\left(f_{a}\right)-F_{a}\left(f_{a}\right)\right|\right)^{\sharp} d x d v d v_{*} d \omega \\
& =\int \chi_{a} B f_{a}^{\prime} f_{a *}^{\prime} F_{j}\left(f_{j *}\right)\left(1-\alpha f_{a}\right)\left(1+(1-\alpha) f_{a}\right)^{1-\alpha}\left|\left(\frac{1}{j}+1-\alpha f_{a}\right)^{\alpha-1}-\left(\frac{1}{a}+1-\alpha f_{a}\right)^{\alpha-1}\right| d x d v d v_{*} d \omega .
\end{aligned}
$$

By Lemma 3.3 and Lemma 3.5, this integral restricted to the set where $\left.1-\alpha f_{a}(t, x, v)\right) \leq \frac{2}{a}$, hence where

$$
\left(1-\alpha f_{a}\right)\left|\left(\frac{1}{j}+1-\alpha f_{a}\right)^{\alpha-1}-\left(\frac{1}{a}+1-\alpha f_{a}\right)^{\alpha-1}\right| \leq 2\left(1-\alpha f_{a}\right)^{\alpha} \leq \frac{2^{\alpha+1}}{a^{\alpha}},
$$

is bounded by $\frac{c}{a^{\alpha}}$ for some constant $c>0$.

For the remaining domain of integration where $\left.1-\alpha f_{a}(t, x, v)\right) \geq \frac{2}{a}$, it holds

$$
\begin{aligned}
\left|F_{j}\left(f_{a}\right)-F_{a}\left(f_{a}\right)\right| & \leq c\left(1-\alpha f_{a}\right)^{\alpha}\left|\left(\frac{1}{j\left(1-\alpha f_{a}\right)}+1\right)^{\alpha-1}-\left(\frac{1}{a\left(1-\alpha f_{a}\right)}+1\right)^{\alpha-1}\right| \\
& =c\left(\frac{1}{j}-\frac{1}{a}\right)\left(1-\alpha f_{a}\right)^{\alpha-1} \lambda^{\alpha-2} \quad \text { where } \lambda \in\left[1, \frac{3}{2}\right] \\
& \leq \frac{2^{\alpha-1} c}{a^{\alpha}} .
\end{aligned}
$$

And so,

$$
\int \chi_{a} B\left(f_{a}^{\prime} f_{a *}^{\prime} F_{j}\left(f_{j *}\right)\left|F_{j}\left(f_{a}\right)-F_{a}\left(f_{a}\right)\right|\right)^{\sharp} d x d v d v_{*} d \omega \leq \frac{c}{a^{\alpha}} .
$$

Finally

$$
\int \chi_{a} B\left(f_{a}^{\prime} f_{a *}^{\prime} F_{j}\left(f_{j *}\right)\left|F_{j}\left(f_{j}\right)-F_{j}\left(f_{a}\right)\right|\right)^{\sharp}(t, x, v) d x d v d v_{*} d \omega \leq c \int\left|F_{j}\left(f_{j}\right)-F_{j}\left(f_{a}\right)\right|^{\sharp}(t, x, v) d x d v .
$$

Split the $(x, v)$-domain of integration of the latest integral into

$$
\begin{aligned}
D_{1} & :=\left\{(x, v) ;\left(f_{j}^{\sharp}(t, x, v), f_{a}^{\sharp}(t, x, v)\right) \in\left[0, \frac{1}{\alpha}-\mu\right]^{2}\right\}, \\
D_{2} & :=\left\{(x, v) ;\left(f_{j}^{\sharp}(t, x, v), f_{a}^{\sharp}(t, x, v)\right) \in\left[\frac{1}{\alpha}-\mu, \frac{1}{\alpha}\right]^{2}\right\}, \\
D_{3} & \left.:=\left\{(x, v) ;\left(f_{j}^{\sharp}, f_{a}^{\sharp}\right)(t, x, v) \in\left[\frac{1}{\alpha}-\mu, \frac{1}{\alpha}\right] \times\left[0, \frac{1}{\alpha}-\mu\right] \text { or }\left(f_{j}^{\sharp}, f_{a}^{\sharp}\right)(t, x, v)\right) \in\left[0, \frac{1}{\alpha}-\mu\right] \times\left[\frac{1}{\alpha}-\mu, \frac{1}{\alpha}\right]\right\} .
\end{aligned}
$$


It holds that

$$
\begin{aligned}
& \int_{D_{1}}\left|F_{j}\left(f_{j}\right)-F_{j}\left(f_{a}\right)\right|^{\sharp}(t, x, v) d x d v \leq c(\alpha \mu)^{\alpha-1} \int_{D_{1}}\left|g_{j}^{\sharp}(t, x, v)\right| d x d v, \\
& \int_{D_{2}}\left|F_{j}\left(f_{j}\right)-F_{j}\left(f_{a}\right)\right|^{\sharp}(t, x, v) d x d v \leq c t^{\alpha-1} \int_{D_{2}}\left|g_{j}^{\sharp}(t, x, v)\right| d x d v, \quad \text { by (4.5), } \\
& \int_{D_{3}}\left|F_{j}\left(f_{j}\right)-F_{j}\left(f_{a}\right)\right|^{\sharp}(t, x, v) d x d v \leq c\left((\alpha \mu)^{\alpha-1}+t^{\alpha-1}\right) \int_{D_{3}}\left|g_{j}^{\sharp}(t, x, v)\right| d x d v .
\end{aligned}
$$

The remaining terms to the right in (4.7) are of the same types as the ones just estimated. Consequently,

$$
\frac{d}{d t} \int\left|g_{j}^{\sharp}(t, x, v)\right| d x d v \leq \frac{c}{a^{\alpha}}+c\left(1+t^{\alpha-1}\right)\left(\int\left|g_{j}^{\sharp}(t, x, v)\right| d x d v\right) .
$$

And so,

$$
\int\left|g_{j}^{\sharp}(t, x, v)\right| d x d v \leq\left(\int_{|v|>a} f_{0}(x, v) d x d v+\frac{c T}{a^{\alpha}}\right) e^{c\left(T+\frac{T^{\alpha}}{\alpha}\right)},
$$

which tends to zero when $a \rightarrow+\infty$, uniformly w.r.t. $j \geq a$. This proves that $\left(f_{j}\right)_{j \in \mathbb{N}^{*}}$ is a Cauchy sequence in $L^{1}\left([0, T] \times[0,1] \times \mathbb{R}^{2}\right)$ and ends the proof of the existence of a solution $f$ to $(2.5)$.

One can similarly prove that the solution is unique and stable. The energy is non-increasing. The conservation of mass and first momentum of $f$ follow from the boundedness of the total energy.

Energy conservation will follow if the energy is non-decreasing. Taking $\psi_{\epsilon}=\frac{\left|v^{2}\right|}{1+\epsilon|v|^{2}}$ as approximation for $|v|^{2}$, it is enough to bound

$$
\int Q(f, f)(t, x, v) \psi_{\epsilon}(v) d x d v=\int B \psi_{\epsilon}\left(f^{\prime} f_{*}^{\prime} F(f) F\left(f_{*}\right)-f f_{*} F\left(f^{\prime}\right) F\left(f_{*}^{\prime}\right)\right) d x d v d v_{*} d \omega
$$

from below by zero in the limit $\epsilon \rightarrow 0$. Now [12]

$$
\begin{aligned}
\int Q(f, f) \psi_{\epsilon} d x d v & =\frac{1}{2} \int B f f_{*} F\left(f^{\prime}\right) F\left(f_{*}^{\prime}\left(\psi_{\epsilon}\left(v^{\prime}\right)+\psi_{\epsilon}\left(v_{*}^{\prime}\right)-\psi_{\epsilon}(v)-\psi_{\epsilon}\left(v_{*}\right)\right) d x d v d v_{*} d \omega\right. \\
& \geq-\int B f f_{*} F\left(f^{\prime}\right) F\left(f_{*}^{\prime}\right) \frac{\epsilon|v|^{2}\left|v_{*}\right|^{2}}{\left(1+\epsilon|v|^{2}\right)\left(1+\epsilon\left|v_{*}\right|^{2}\right)} d x d v d v_{*} d \omega .
\end{aligned}
$$

The previous line, with the integral taken over a bounded set in $\left(v, v_{*}\right)$, converges to zero when $\epsilon \rightarrow 0$. In integrating over $|v|^{2}+\left|v_{*}\right|^{2} \geq 2 \lambda^{2}$, there is symmetry between the subset of the domain with $|v|^{2}>\lambda^{2}$ and the one with $\left|v_{*}\right|^{2}>\lambda^{2}$. We discuss the first sub-domain, for which the integral in the last line is bounded from below by

$-c \int\left|v_{*}\right|^{2} f\left(t, x, v_{*}\right) d x d v_{*} \int_{|v| \geq \lambda} B \sup _{(s, x) \in[0, t] \times[0,1]} f^{\#}(s, x, v) d v d \omega \geq-c \int_{|v| \geq \lambda} \sup _{0 \leq s, x \in[0,1]} f^{\#}(s, x, v) d v$.

It follows from Lemma 4.2 that the right hand side tends to zero when $\lambda \rightarrow \infty$. This implies that the energy is non-decreasing, and bounded from below by its initial value. That completes the proof of the theorem. 


\section{References}

[1] L. Arkeryd, A quantum Boltzmann equation for Haldane statistics and hard forces; the spacehomogeneous initial value problem, Comm. Math. Phys., 298 (2010), 573-583.

[2] R. K. Bhaduri, R. S. Bhalerao, M. V. Murthy, Haldane exclusion statistics and the Boltzmann equation, J. Stat. Phys., 82 (1996), 1659-1668.

[3] R. K. Bhaduri, M. V. Murthy, M. Brack, Fermionic ground state at unitarity and Haldane exclusion statistics, J. Phys. B, 41 (2008), 115301.

[4] J.-M. Bony, Solutions globales bornées pour les modèles discrets de l'équation de Boltzmann, en dimension 1 d'espace, in: Journées "Équations aux dérivées partielles ", Exp. XVI, École Polytech. (1987), Palaiseau, 1-10.

[5] C.Cercignani, R. Illner, Global weak solutions of the Boltzmann equation in a slab with diffusive boundary conditions, Arch Rat. Mech. Anal. 134 (1996), 1-16.

[6] J. Dolbeault, Kinetic models and quantum effects: a modified Boltzmann equation for FermiDirac particles, Arch. Rat. Mech. Anal. 127 (1994), 101-131.

[7] M. Escobedo, S. Mischler, M. Valle, Homogeneous Boltzmann equation in quantum relativistic kinetic theory, Electronic J. Diff. Eqns., Monograph 04 (2003).

[8] F. D. Haldane, Fractional statistics in arbitrary dimensions: a generalization of the Pauli principle, Phys. Rev. Lett. 67 (1991), 937-940.

[9] J. M. Leinaas, J. Myrheim, On the theory of identical particles, Nuovo Cim. B N.1 (1977), $1-23$.

[10] P. L. Lions, Compactness in Boltzmann's equation via Fourier integral operators and applications I, III, J. Math. Kyoto Univ.,34 (1994), 391-427, 539-584.

[11] X. Lu, A modified Boltzmann equation for Bose-Einstein particles: isotropic solutions and long time behaviour, J. Stat. Phys., 98 (2000), 1335-1394.

[12] X. Lu, On isotropic distributional solutions to the Boltzmann equation for Bose-Einstein particles, J. Stat. Phys. 116 (2004), 1597-1649.

[13] X. Lu, The Boltzmann equation for Bose-Einstein particles: velocity concentration and convergence to equilibrium, J. Stat. Phys. 119 (2005), 1027-1067.

[14] Lu, X., The Boltzmann equation for Bose-Einstein particles: condensation in finite time, J. Stat. Phys. 150 (2013), 1138-1176. 
[15] L. W. Nordheim, On the kinetic methods in the new statistics and its applications in the electron theory of conductivity, Proc. Roy. Soc. London Ser. A 119, 689-698 (1928).

[16] G. Royat, Etude de l'équation d'Uehling-Uhlenbeck: existence de solutions proches de Planckiennes et étude numérique, Thèse, Marseille 2010.

[17] Y. S. Wu, Statistical distribution for generalized ideal gas of fractional-statistics particles, Phys. Rev. Lett. 73 (1994), 922-925. 